\title{
samrnat of Vascular Research
}

C. Aalkjaer, Aarhus

P.W. Aaronson, London

T.H. Adair, Jackson, MS

L.P. Adam, Princeton, NJ

S. Aharinejad, Vienna

J.S. Alexander, Shreveport, LA

K. Alitalo, Helsinki

H.R. Andersen, Aarhus

W.P. Anderson, Clayton

R. Andriantsitohaina, Illkirch

J.A. Angus, Victoria

H.G. Augustin, Freiburg

T.Y. Aw, Shreveport, LA

D. Axel, Tübingen

U. Baandrup, Aarhus

J.B. Bassingthwaighte, Seattle, WA

D. Bates Bristol

K. Bayless, College Station, TX

B.F. Becker, Munich

J.N. Benoit, Mobile, AL

M. Boegehold, Morgantown, WV

G. Bohlen, Indianapolis, IN

M. Bohm, Homburg/Saar

T.B. Bolton, London

S. Bolz, Munich

J.E. Brayden, Burlington, VT

G. Breier, Bad Nauheim

P.R. Brink, Stony Brook, NY

R.M. Bryan, Houston, TX

G. Burnstock, London

H. Burri, Bern

A.E. Canfield, Manchester

P. Carmeliet, Leuven

O. Carpon, Helsinki

O. Carretero, Detroit, MI

S.A. Carter, Winnipeg

B. Cercek, Los Angeles, CA

G. Christ, Bronx, NY

K.L. Christensen, Aarhus

E. Clauser, Paris

G. Clough, Southampton

J.R. Cockcroft, Cardiff

A.R. Cohen, Boston, MA

J.N. Cohn, Minneapolis, MN

The Editors and the members of the editorial board would like to thank the following individuals for their expert assistance in acting as reviewers for the Journal of Vascular Research in the period of September 1, 2000 to August 31, 2001. Our aim of establishing the journal as a leading journal in the field of vascular science is to a large extent in the hands of our reviewers and we, together with our authors and readers, much appreciate the time which they have freely given.

T. Cornwell, Birmingham, AL

F.E. Curry, Davis, CA

M.D. Dake, Stanford, CA

D.H. Damon, Burlington, VT

P.A. D'Amore, Boston, MA

J. Danser, Rotterdam

H.E. D'Arceuil, Charlestown, MA

G. Davis, College Station, TX

R. Dechend, Berlin

D.J. de Wildt, Utrecht

C. de Witt, Munich

S. Diamond, Philadelphia, PA

H.H. Dietrich, St. Louis, MO

N. Dietz, Columbia, MO

J. Diez, Pamplona

K.A. Dora, Bath

H. Drexler, Hannover

B. Duling, Charlottesville, VA

W.N. Duran, Newark, NJ

N. Eigler, Los Angeles, CA

B. Engelmann, Munich

B. Fagrell, Stockholm

N. Ferrara, San Francisco, CA

C. Ferrario, Winston Salem, NC

W.R. Ferrell, Glasgow

J.A. Firth, London

T.A. Fischell, Kalamazoo, MI

R. Forough, College Station, TX

P. Fraser, London

J.C. Frisbee, Milwaukee, WI

K. Fujiwara, Rochester, NY

G. Gabbiani, Geneva

J. Galle, Würzburg

S.M. Gardiner, Nottingham

T.W. Gardner, Hershey, PA

D. Gebremedhin, Milwaukee, WI

W.T. Gerthofer, Reno, NV

H. Gerwitz, Boston, MA

X. Girerd, Paris

T. Gloe, Munich

P. Goetinck, Charlestown, MA

D.N. Granger, Shreveport, LA
S. Greenwald, London

K.K. Griendling, Atlanta, GA

T.M. Griffith, Cardiff

D.D. Gutterman, Milwaukee, WI

H. Hacker, Munich

J.R. Haeberle, Burlington, TX

G. Hamann, Munich

P. Hamet, Montréal

G.K. Hansson, Stockholm

B. Haraldsson, Göteborg

D.G. Harrison, Atlanta, GA

M. Hasenkam, Aarhus

C.C. Haudenschild, Rockville, MD

J.R. Hawker, Temple, TX

S. Haworth, London

D. Hayoz, Lausanne

M.A. Head, Bundoora

J.P. Headrick, Brisbane

M. Hecker, Göttingen

C.C. Hedrick, Charlottesville, VA

T. Hein, College Station, TX

A. Heinemann, Graz

B. Hennig, Lexington, KY

R.L. Hester, Jackson, MS

G. Heusch, Essen

T. Higenbottam, Sheffield

M. Hill, Bundoora

B. Hillen, Utrecht

T. Hintze, Valhalla,

G.D. Hirst, Reno, NV

A.P.G. Hoeks, Maastricht

J.J. Holst, Copenhagen

V. Howard, Liverpool

P.L. Huang, Charleston, MA

O. Hudlicka, Birmingham

J.D. Humphrey, College Station, TX

J.D. Imig, New Orleans, LA

T. Inagami, Nashville, TN

E.W. Inscho, Augusta, GA

M. Intaglietta, La Jolla, CA

W.F. Jackson, Kalamazoo, MI

R. Jalan, London

L.J. Jansson, Hamilton 
R.A. Johns, Baltimore, MD

J.M. Johnson, San Antonio, TX

J. Jordan, Berlin

J.J. Joyner, Rochester, NY

F. Jung, Dresden

M. Jünger, Tübingen

G. Kaley, Valhalla, NY

V.K. Kalra, Los Angeles, CA

Z.S. Katusic, Rochester, MN

J.L. Kelley, Johnson City, TN

D.P. Kelly, St. Louis, MO

R. Kenagy, Seattle, WA

D. Kirsch, Mainz

V. Kolb-Backofen, Düsseldorf

A. Koller, Valhalla, NY

R.J. Korthuis, Shreveport, LA

J.M. Krum, Washington, DC

P. Kubes, Calgary

L. Kuo, College Station, TX

P. Lacolley, Paris

V. Lahera, Madrid

D. Lamontagne, Montréal

H. Landgraf, Berlin

U. Laufs, Homburg

S. Laurent, Paris

T. Länne, Jönköping

R.M.K.W. Lee, Hamilton

H.A. Lehr, Mainz

F. Le Noble, Paris

H. Leonhardt, Berlin

B. Lévy, Paris

K. Ley, Charlottesville, VA

U. Lindauer, Berlin

C.D. Little, Kansas City, KS

S.Q. Liu, Evanston, IL

J.H. Lombard, Milwaukee, WI

R. Loutzenhiser, Calgary

F.C. Luft, Berlin

M. Lundberg, Bethesda, MD

A. Lückhoff, Aachen

J.S. Lymn, London

F. Mahler, Bern

L. Martinez, College Station, TX

S. Maxwell, College Station, TX

W.G. Mayhan, Omaha, NE

H.N. Mayrovitz, Ft. Lauderdale, FL

P.F. McDonagh, Flagstaff, AZ

L.V. McIntire, Houston, TX

J. McMilin, Houston, TX

C. Meininger, Temple, TX

K. Messmer, Munich

R. Mestril, Maywood, IL

J.M. Miano, Milwaukee, WI

C.C. Michel, Alderney

J.-B. Michel, Paris

A.W. Miller, Winston Salem, NC

V.M. Miller, Rochester, MN
H. Mischak, Hannover

R.S. Moreland, Philadelphia, PA

E. Morkin, Tuscon, AZ

L. Musil, Portland, OR

T. Münzel, Pinneberg

O.A. Nedergaard, Odense

S. Nees, Munich

T.O. Neild, Bedford Park

A.C. Newby, Bristol

B. Nilius, Leuven

H. Nilsson, Aarhus

J.R. Nyengaard, Aarhus

J. Ohanian, Manchester

H. Olschewski, Giessen

R.A. Olsson, Charlottesville, VA

C. O'Neill, Atlanta, GA

S. Oparil, Birmingham

M. O'Rourke, Sydney

O.N. Osipenko, Glasgow

M. Oude Egbrink, Maastricht

P. Parsons-Wingerter, San Francisco, CA

G. Pasterkamp, Utrecht

R.A. Peattie, Salt Lake City, UT

H. Pessenhofer, Graz

J. Pfeilschifter, Frankfurt

S. Pfister, Milwaukee, WI

M.I. Philips, Gainesville, FL

B.R. Pitt, Pittsburg, PA

S. Platts, Charlottesville, VA

M.J. Post, Boston, MA

J.F. Potter, Leicester

J.T. Powell, London

R.J. Powell, Lebanon, NH

E.W. Raines, Seattle, WA

K.S. Ramos, College Station, TX

T.G. Redgrave, Crawley

R.K. Reed, Bergen

D. Ribatti, Bari

B. Rippe, Lund

B. Risberg, Göteborg

J.M. Ritter, London

D. Rizzoni, Brescia

L.J. Roberts, Nashville, TN

J. Röther, Hamburg

J.M. Rubin, Ann Arbor, MI

T.J. Ryan, Oxford

M. Safar, Paris

S. Sartore, Padova

M. Schachter, London

K. Schmidt, Graz

D. Schneider, Colchester, VT

J.D. Schulzke, Berlin

R.S. Schwartz, Rochester, MN

T. Scott-Burden, Houston, TX

N.H. Secher, Copenhagen
C. Seidel, Houston, TX

N.J. Severs, London

H. Shimokawa, Fukuoka

H. Singer, Albany, NY

J.J. Sixma, Utrecht

J.V. Small, Salzburg

E.J. Smart, Lexington, KY

J. Smith, Exeter

L. Snoeckx, Maastricht

J.A.E. Spaan, Amsterdam

W.B. Stallcup, La Jolla, CA

C.T. Stier, Valhalla, NY

M.S. Sturek, Columbia, MO

D. Sugden, London

A. Surprenant, Sheffield

W.R. Taylor, Atlanta, GA

H. Thorlacius, Malmö

C. Thuillez, Rouen

C. Tiruppathi, Chicago, IL

R.J. Tomanek, Iowa City, IA

R. Touyez, Québec

R. Traystmann, Baltimore, MD

M. Ursino, Bologna

P. Vajkoczy, Mannheim

L.M. van Bortel, Ghent

C. van Breemen, Vancouver

W.H. Van Gilst, Groningen

P.M. Vanhoutte, Courbevoie

R.J. van Suylen, Maastricht

L. Varticovski, Boston, MA

A. Veves, Boston, MA

J. Vinten-Johansen, Atlanta, GA

M.M. Voigt, St. Louis, MO

B. Vollmar, Homburg/Saar

P. Walsh, Calgary

J.P.T. Ward, London

R.C. Webb, Augusta, GA

C. Weber, München

P.D. Weinberg, Reading

A.H. Weston, Manchester

R.E. Widdop, Victoria

K. Willecke, Bonn

B. Williams, Leicester

E. Wilson, College Station, TX

V.G. Wilson, Nottingham

J. Wilting, Freiburg

O.L. Woodman, Melbourne

G. Wu, College Station, TX

G.D. Yancopoulos, Tarrytown, NY

H.-G. Zimmer, Leipzig 